\title{
Sonication of Removed Breast Implants for Improved Detection of Subclinical Infection
}

\author{
U. M. Rieger · G. Pierer · N. J. Lüscher · \\ A. Trampuz
}

Received: 7 January 2009/ Accepted: 20 February 2009/Published online: 26 March 2009

(C) Springer Science+Business Media, LLC and International Society of Aesthetic Plastic Surgery 2009

\begin{abstract}
Background Capsular fibrosis is a severe complication after breast implantation with an uncertain etiology. Microbial colonization of the prosthesis is hypothesized as a possible reason for the low-grade infection and subsequent capsular fibrosis. Current diagnostic tests consist of intraoperative swabs and tissue biopsies. Sonication of removed implants may improve the diagnosis of implant infection by detachment of biofilms from the implant surface.

Methods Breast implants removed from patients with Baker grades 3 and 4 capsular contracture were analyzed by sonication, and the resulting sonication fluid was quantitatively cultured.

Results This study investigated 22 breast implants (6 implants with Baker 3 and 16 implants with Baker 4 capsular fibrosis) from 13 patients. The mean age of the patients was 49 years (range, 31-76 years). The mean
\end{abstract}

\section{U. M. Rieger · G. Pierer}

Department of Plastic, Reconstructive, and Aesthetic Surgery, University Hospital of Basel, Spitalstrasse 21, 4031 Basel, Switzerland

\section{U. M. Rieger $(\bowtie) \cdot$ G. Pierer}

Department of Plastic, Reconstructive, and Hand Surgery, Innsbruck Medical University, Anichstrasse 35,

6020 Innsbruck, Austria

e-mail: ulrich.rieger@uki.at; riegeru@uhbs.ch

\section{N. J. Lüscher}

Plastic Surgery Unit, Crossklinik and Merian Iselin Spital, Swiss Olympic Medical Center, Föhrenstrasse 2,

4009 Basel, Switzerland

\section{A. Trampuz}

Division of Infectious Diseases and Hospital Epidemiology, Department of Internal Medicine, University Hospital of Basel, Petersgraben 4, 4031 Basel, Switzerland implant indwelling time was 10.4 years (range, 3 months to 30 years). Of the 22 implants, 12 were used for breast reconstruction and 10 for aesthetic procedures. The implants were located subglandularly $(n=12)$, submuscularly $(n=6)$, and subcutaneously $(n=4)$. Coagulasenegative staphylococci, Propionibacterium acnes, or both were detected in the sonication fluid cultures of nine implants (41\%), eight of which grew significant numbers of microorganisms (>100 colonies $/ \mathrm{ml}$ of sonication fluid). Conclusions Sonication detected bacteria in $41 \%$ of removed breast implants. The identified bacteria belonged to normal skin flora. Further investigation is needed to determine any causal relation between biofilms and capsular fibrosis.

Keywords Biofilm - Breast implant - Capsular fibrosis . Contracture $\cdot$ Sonication $\cdot$ Subclinical infection

Periprosthetic capsular contracture is a severe complication experienced by $30 \%$ of patients after breast prosthesis implantation [5-7]. The etiology of capsular contracture remains unclear. Implant filling, placement of the prosthesis, surface texture, and low-grade prosthesis infection are hypothesized as influencing the formation of capsular fibrosis and subsequent contracture [4].

We specifically investigated microbial colonization of the implant as the possible cause for a persistent chronic low-grade infection and subsequent formation of capsular fibrosis, as previously suggested by other investigators $[7-9,11]$. This hypothesis is supported by the fact that unilateral contractures may occur after bilateral augmentation surgery with identical implants, making systemic (host-related) and implant-specific causes less likely. 
Several studies aimed to detect adherent bacteria growing in biofilms on breast implants, but no conclusive data were published. Most of these studies used conventional swabs, biopsies of the periprosthetic fibrotic capsule, or both, and judged the results to be positive if at least one of several samples yielded bacterial growth [2, 12]. This microbiologic method, however, lacks sensitivity and specificity, as demonstrated in other surgical specialties such as orthopedic surgery [3]. Conventional swabs can be false-negative in about $30 \%$ of cases with prosthetic joint infection, making this method unreliable for detecting implant-associated infection.

Sonication of removed implants is a new diagnostic method shown by our group to improve the diagnosis of prosthetic joint infection significantly by detachment of microbial biofilms from the hip and knee prosthetic surface [14]. Furthermore, sonication of parts of breast implants and capsule biopsies performed by Pajkos et al. [9] yielded positive cultures for $38.5 \%$ of implants and $89.5 \%$ of capsules involving severely contracted breasts. We hypothesized that sonication of whole-breast implants with an optimized sonication method can improve the detection of microbial colonization of removed breast implants and generate new insights into the pathogenesis of capsular contracture.

\section{Patients and Methods}

\section{Study Population}

The study was conducted at the University Hospital Basel, Switzerland, an 800-bed primary and tertiary health care center. This hospital is the major provider of acute medical care for about 300,000 inhabitants. The study enrolled patients undergoing breast implant removal for Baker 3 and 4 capsular contracture in the Department of Plastic, Reconstructive, and Aesthetic Surgery at the University Hospital Basel, Switzerland between March 2007 and February 2008. Patients were excluded if obvious contamination occurred in the operating room.

\section{Collection of Breast Implants}

Whole-breast implants were aseptically removed from the patient, and each was placed in a separate sterile polyethylene container (Lock \& Lock, HPL 933; Vetrag AG, Stäfa, Switzerland). The reason for implant removal, the implant type and placement, and the indwelling time of the implant were recorded by the surgeon. The surgeon assessed breast firmness using the Baker manual scaling method [13].

\section{Sonication of Breast Implants}

In the microbiologic laboratory, $100 \mathrm{ml}$ of sterile Ringer's solution was added to each container holding the breast implant, which was processed within $6 \mathrm{~h}$ of removal (Fig. 1). The container was vortexed for $30 \mathrm{~s}$, then sonicated for $1 \mathrm{~min}$ at a frequency of $40 \pm 2 \mathrm{kHz}$ and a power density of $0.22 \pm 0.04 \mathrm{~W} / \mathrm{cm}^{2}$, as determined by a calibrated hydrophone (Type 8103; Brüel and Kjær, Naerum, Denmark).

For sonication, an ultrasound bath (BactoSonic; Bandelin $\mathrm{GmbH}$, Berlin, Germany) was used. The resulting sonication fluid was plated in aliquots of $0.1 \mathrm{ml}$ onto aerobic and anaerobic sheep blood agar plates, incubated at $37^{\circ} \mathrm{C}$ for 7 days, and inspected daily for bacterial growth. Microorganisms were enumerated and classified using routine microbiologic techniques. Positive sonication was considered if 10 colony-forming units (CFU)/ml or more of sonication fluid were detected.

Negative Control Implants

Three sterilized breast implants were included in the sonication process as negative control implants. These were placed on the table with the surgical instruments in the regular operating room. They subsequently were placed in sterile polyethylene containers, then processed as described earlier for implants collected from patients.

\section{Results}

\section{Patient Characteristics}

During the study period, 22 breast implants from 13 patients were investigated (Table 1). At the time of implant removal, the mean patient age was 49 years (range, 31-76 years). For

Fig. 1 Sonication procedure. Aseptically removed breast implants were placed in sterile containers, then vortexed and sonicated in Ringer's solution

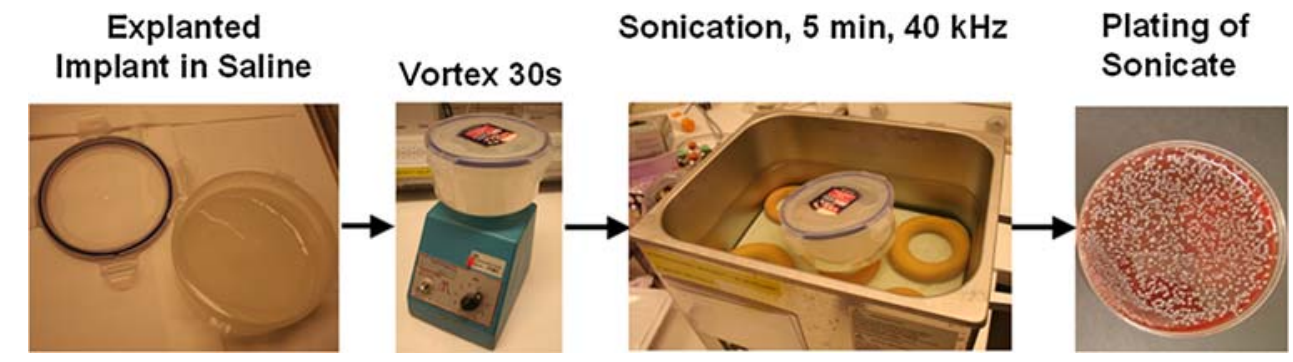


Table 1 Patient characteristics

\begin{tabular}{lc}
\hline Characteristic & Total $(n=22)$ \\
\hline Age (years): mean (range) & $49(31-76)$ \\
Implant indwelling time (years): mean (range) & $10.4(0.25-30)$ \\
Preexisting breast disease: $n(\%)$ & \\
$\quad$ Breast hypoplasia (aesthetic procedure) & $12(55)$ \\
$\quad$ Breast cancer (reconstructive procedure) & $10(45)$ \\
Capsular contracture: $n(\%)$ & \\
Baker 3 & $6(27)$ \\
Baker 4 & $16(73)$ \\
\hline
\end{tabular}

4 implants (18\%), previous implant replacement surgeries had been performed before the current surgical procedure due to capsular contracture, whereas for 18 implants (82\%), the current surgery was the first procedure after primary breast implantation. The mean implant indwelling time was 10.4 years (range, 3 months to 30 years). Of the 22 implants, $12(55 \%)$ were used for breast reconstruction and $10(45 \%)$ for aesthetic procedures. At explantation, the diagnosis was Baker 3 capsular contracture for 6 implants (27\%) and Baker 4 contracture for 16 implants (73\%).

\section{Implant Characteristics}

In terms of placement, 12 implants were positioned subglandularly, 6 submuscularly (partially), and 4 subcutanously (all reconstructions only) (Table 2). The surface structure was textured for 16 implants (73\%) and smooth

Table 2 Implant characteristics and relative sonication results

\begin{tabular}{|c|c|c|}
\hline Characteristic & $\begin{array}{l}\text { Total }(n=22) n \\
(\%)\end{array}$ & $\begin{array}{l}\text { Positive sonication } \\
\text { results }(n=9) n(\%)\end{array}$ \\
\hline \multicolumn{3}{|l|}{ Location of implant } \\
\hline Subglandular & $12(55)$ & $5 / 12(42)$ \\
\hline Subpectoral & $6(27)$ & $3 / 6(50)$ \\
\hline $\begin{array}{l}\text { Subcutanous } \\
\text { (reconstructive) }\end{array}$ & $4(18)$ & $1 / 4(25)$ \\
\hline \multicolumn{3}{|l|}{ Surface of implant } \\
\hline Textured & $16(73)$ & $7 / 16(44)$ \\
\hline Smooth & $6(27)$ & $2 / 6(33)$ \\
\hline \multicolumn{3}{|l|}{ Type of implant } \\
\hline $\begin{array}{l}\text { Silicone gel (high } \\
\text { cohesive) }\end{array}$ & $20(91)$ & $9 / 20(45)$ \\
\hline $\begin{array}{l}\text { Silicone liquid (low } \\
\text { cohesive) }\end{array}$ & $2(9)$ & $0 / 2(0)$ \\
\hline \multicolumn{3}{|l|}{ Volume of implant (ml) } \\
\hline$<250$ & $13(59)$ & $6 / 13(46)$ \\
\hline$\geq 250$ & $5(23)$ & $3 / 5(60)$ \\
\hline Ruptured & $4(18)$ & $0 / 4(0)$ \\
\hline
\end{tabular}

for 6 implants (27\%). Of the 22 implants, 20 contained silicone gel (high cohesive), and 2 contained silicone fluid (low cohesive). The mean volume of the breast implants was $230 \mathrm{ml}$ (range, 130-750 ml).

\section{Microbiology}

Nine $(41 \%)$ of the implants showed significant numbers of bacteria growing in sonication fluid cultures $(>10 \mathrm{CFU} / \mathrm{ml}$ of sonication fluid). Coagulase-negative staphylococci were identified on three implants, Propionibacterium acnes on two implants, and both organisms (coagulase-negative staphylococci and Propionibacterium acnes) on four implants. On 8 (89\%) of 9 implants with a positive sonication culture, high numbers of microorganisms ( $>100 \mathrm{CFU} /$ $\mathrm{ml}$ of sonication fluid) were detected, indicating a multiplelayer biofilm on the prosthesis surface.

\section{Negative Controls}

None of the three sterile implants investigated by sonication showed any growth in the sonication fluid culture.

\section{Conjoint Analysis of Implant Characteristics} and Microbiology

The colonization rates for implant surfaces with regard to location of the implant (subglandular, submuscular, subcutaneous placement), implant surface (smooth vs textured), type of implant (liquid [low cohesive] vs gel [high cohesive] silicone), and implant volume ( $\geq 250$ vs $<250$ $\mathrm{ml}$ ) are shown in Table 2. No significant differences in colonization rates with biofilms were detected among the aforementioned groups.

\section{Discussion}

Clinically manifested postoperative infection after breast implant placement is rare [1]. However, considering the hypothesis that subclinical infection plays a role in the development of capsular fibrosis, the incidence of bacterial colonization on breast implants may be much higher [7, $9,10]$.

The bacteria identified in our study consisted exclusively of skin flora. This finding has been confirmed by others [9, 12]. Previous studies had failed to detect bacteria consistently using swabs and tissue biopsies. The cultures tested positive in $30 \%$ to $67 \%$ of cases [12]. It is difficult to interpret the results of these studies because a single swab or biopsy was considered positive, which may have represented contamination $[2,12]$. 
Pajkos et al. [9] have described the use of a sonication method to detect biofilms on parts of breast implants and on fibrotic capsule biopsies. This method yielded positive culture results for 24 (50\%) of 48 cases including Baker 1 and 2 contractures. In the analysis of Pajkos' subgroups (Baker 3 and 4 capsular contractures), the cultures of capsule samples tested positive in $89 \%$ of cases, whereas the implant pieces tested positive in $38.5 \%$ of cases.

The better sensitivity of sonication in detecting subclinical infection and biofilms on orthopedic implants compared with standard swabs and biopsies has been confirmed by our group [14]. Because of the high sensitivity, we aimed to apply the sonication method only to implants causing Baker 3 and 4 capsular contracture because these contractures yielded the highest numbers of positive cultures in studies using conventional microbiology $[2,12]$.

We found that $41 \%$ of implants showed significant numbers of bacteria on the prosthesis surface. These results in our series may seem inferior to the findings of Pajkos et al. [9] and those of other studies yielding up to 67\% of colonized implants in patients with Baker 3 and 4 capsular contractures. However, these results often were determined from only one positive swab or biopsy, which may represent contamination, especially when bacteria from the skin flora are involved. Therefore, in orthopedic surgery, at least two tissue specimens must test positive before the results are considered positive for low-virulent organisms. The same holds true for blood cultures [3].

In addition, our sonication method allows quantification of recovered bacteria in the sonication fluid, which can distinguish between contamination during prosthesis removal and biofilm infection of breast implants. Pajkos et al. [9] quantified bacteria in seven samples from three patients. In these seven samples, the numbers of bacterial counts were highly variable. Therefore, in our opinion, an accurate distinction between infection, typically comprising a multilayer of biofilm, and a contamination, typically involving low numbers of bacteria belonging to skin flora, is possible only by quantifying the numbers of removed bacteria after sonication. Our approach intended to avoid sampling errors that may occur when portions of the implant are sonicated instead of the whole implant.

Our study had some limitations, including a lack of sonication of capsules (parts or complete), which may detect bacteria in the culture for patients with Baker 3 and 4 capsular contractures. The sensitivity of the sonication method could have been gained if whole capsules had been sonicated. However, extirpation of whole capsules often may not be feasible or desired from a surgical perspective. In most cases, whole capsules were not available to us. Sensitivity would be improved further if sonication for breast implants were individually optimized because the acoustic parameters may not be transferable directly from mechanical orthopedic implants to breast implants due to differences in material, surface structure, and density.

\section{Conclusion}

Sonication of whole implants may be a useful addition to the armamentarium of procedures used to detect microorganisms on breast implants. In our series, bacteria were detected in $41 \%$ of removed breast implants. The identified bacteria belonged to normal skin flora, which colonized the implant either during implantation or later by lymphogenous or hematogenous spread or by lactiferous duct contamination. Further investigation is needed to determine the causal relation between biofilms and capsular fibrosis.

Acknowledgments Part of this work was presented at the Annual Meeting of the Swiss Society of Plastic Reconstructive and Aesthetic Sugery (SGPRAC) in Montreux, Switzerland, September 2007. The presentation was elected to represent Switzerland in the "Best of Europe" Session for the Annual Meeting of the European Association of Societies of Aesthetic Plastic Surgery (EASAPS).

\section{References}

1. Araco A, Gravante G, Araco F, Delogu D, Cervelli V, Walgenbach K (2007) Infections of breast implants in aesthetic breast augmentations: a single-center review of 3,002 patients. Aesth Plast Surg 31:325-329

2. Burkhardt BR, Fried M, Schnur PL, Tofield JJ (1981) Capsules, infection, and intraluminal antibiotics. Plast Reconstr Surg 68:43-49

3. Darouiche RO (2004) Treatment of infections associated with surgical implants. N Engl J Med 350:1422-1429

4. Embrey M, Adams EE, Cunningham B, Peters W, Young VL, Carlo G (1999) A review of the literature on the etiology of capsular contracture and a pilot study to determine the outcome of capsular contracture interventions. Aesth Plast Surg 23:197206

5. Ersek RA (1991) Rate and incidence of capsular contracture: a comparison of smooth and textured silicone double-lumen breast prostheses. Plast Reconstr Surg 87:879-884

6. Ersek RA, Salisbury AV (1997) Textured surface, nonsilicone gel breast implants: four years' clinical outcome. Plast Reconstr Surg 100:1729-1739

7. Gabriel SE, Woods JE, O'Fallon WM, Beard CM, Kurland LT, Melton LJ 3rd (1997) Complications leading to surgery after breast implantation. N Engl J Med 336:677-682

8. Netscher DT (2004) Subclinical infection as a possible cause of significant breast capsules. Plast Reconstr Surg 113:2229-2230

9. Pajkos A, Deva AK, Vickery K, Cope C, Chang L, Cossart YE (2003) Detection of subclinical infection in significant breast implant capsules. Plast Reconstr Surg 111:1605-1611

10. Pittet B, Montandon D, Pittet D (2005) Infection in breast implants. Lancet Infect Dis 5:94-106

11. Ransjo U, Asplund OA, Gylbert L, Jurell G (1985) Bacteria in the female breast. Scand J Plast Reconstr Surg 19:87-89

12. Schreml S, Heine N, Eisenmann-Klein M, Prantl L (2007) Bacterial colonization is of major relevance for high-grade capsular 
contracture after augmentation mammaplasty. Ann Plast Surg 59:126-130

13. Spear SL, Baker JL Jr (1995) Classification of capsular contracture after prosthetic breast reconstruction. Plast Reconstr Surg 96:1119-1123
14. Trampuz A, Piper KE, Jacobson MJ, Hanssen AD, Unni KK, Osmon DR, Mandrekar JN, Cockerill FR, Steckelberg JM, Greenleaf JF, Patel R (2007) Sonication of removed hip and knee prostheses for diagnosis of infection. N Engl J Med 357:654-663 